05,06

\title{
Теория инверсного магнитоэлектрического эффекта в слоистых магнитострикционно-пьезоэлектрических структурах
}

\author{
( Д.А. Филиппов ${ }^{1}$, Г.С. Радченко ${ }^{2,3}$, T.О. Фирсова ${ }^{1}$, Т.А. Галкина ${ }^{1}$ \\ ${ }^{1}$ Новгородский государственный университет им. Ярослава Мудрого, \\ Великий Новгород, Россия \\ ${ }^{2}$ Южный федеральный университет, \\ Ростов-на-Дону, Россия \\ ${ }^{3}$ Научно-исследовательский институт фризики Южного федерального университета, \\ Ростов-на-Дону, Россия \\ E-mail: Dmitry.Filippov@novsu.ru \\ (Поступила в Редакцию 4 июля 2016 г. \\ В окончательной редакции 4 октября 2016 г.)
}

\begin{abstract}
Представлена теория инверсного магнитоэлектрического эффекта в слоистых структурах, основанная на решении уравнений эластодинамики и электростатики отдельно для магнитострикционной и пьезоэлектрической фаз с учетом условий на границе раздела между фазами. Получены выражения для коэффициента инверсного магнитоэлектрического преобразования через параметры, характеризующие магнитострикционную и пьезоэлектрическую фазы. Рассчитаны теоретические зависимости коэффициента инверсного магнитоэлектрического преобразования от частоты переменного электрического поля для трехслойной структуры PZT-Ni-PZT и двухслойной структуры $D$-терфенол-PZT. Результаты расчетов хорошо совпадают с экспериментальными данными.
\end{abstract}

Работа выполнена при частичной поддержке Министерства образования и науки РФ в рамках проектной части государственного задания проект № 177 и гранта конкурса РФФИ-БРФФИ проект № 16-52-00184 Бел_а.

DOI: 10.21883/FTT.2017.05.44371.272

\section{1. Введение}

Магнитоэлектрический (МЭ) эффект, теоретически предсказанный $[1,2]$ и экспериментально обнаруженный $[3,4]$ более полувека назад, в последнее время привлекает все большее число исследователей, о чем говорит рост числа публикаций на эту тему [5]. Уникальность этого эффекта заключается том, что он относится к так называемым перекрестным эффектам и связывает между собой векторы, имеющие различные трансформационные свойства - аксиальный вектор с полярным и, наоборот, полярный с аксиальным. Так, при помещении МЭ-материала в магнитное поле (аксиальный вектор) происходит изменение поляризации (полярный вектор) и, наоборот, при помещении магнитоэлектрика в электрическое поле (полярный вектор) происходит изменение намагниченности (аксиальный вектор). Изменение электрической поляризации под действием магнитного поля получило название прямой МЭ-эффект, а изменение намагниченности под действием электрического поля обычно называют инверсным (конверсным) или обратным МЭ-эффектом. Инверсный МЭ-эффект интересен тем, что позволяет управлять магнитными свойствами вещества электрическим полем, что значительно упрощает конструкцию управляющих устройств, заменяя катушки индуктивности обкладками конденсаторов. Например, в СВЧ-области спектра в МЭ-кристаллах можно при помощи электрического поля управлять сдвигом линии магнитного резонанса [6-9], что позволяет создавать на этой основе такие приборы, как СВЧи фазовращатели, фильтры, аттенюаторы, линии задержки и т.д. Несмотря на многочисленные исследования, выполненные на монокристаллах, широкого применения на практике МЭ-эффект не нашел. С одной стороны, это объясняется малостью величины эффекта, с другой стороны, тем, что в большинстве материалов МЭ-эффект наблюдается при температурах значительно ниже комнатной. Малость МЭ-эффекта в монокристаллах объясняется тем, что механизмом его возникновения является совместное действие нечетной части потенциала внутрикристаллического поля, внешнего электрического поля и спин-орбитального взаимодействия [10]. Спинорбитальное взаимодействие является релятивистским эффектом, чем и объясняется малость МЭ-эффекта в монокристаллах. Ситуация коренным образом изменилась после синтеза магнитоэлектрических композиционных материалов [11,12]. Величина МЭ-эффекта эвтектической композиции $\mathrm{Fe}-\mathrm{Co}-\mathrm{Ti}-\mathrm{Ba}-\mathrm{O}$, полученной методом направленной кристаллизации и представляющей собой чередующиеся слои магнитной шпинели и пьезоэлектрического перовскита, на два порядка превышала величину МЭ-эффекта в монокристалле $\mathrm{Cr}_{2} \mathrm{O}_{3}$. В настоящее время синтезировано достаточно много различных композиционных магнитоэлектрических материалов, величина эффекта в которых достаточна для практического применения $[13,14]$. 
Механизмом возникновения МЭ-эффекта в композиционных материалах является механическое взаимодействие магнитострикционной и пьезоэлектрической подсистем. При прямом эффекте в переменном магнитном поле в магнитострикционной компоненте возникают деформации, которые посредством механического взаимодействия передаются в пьезоэлектрическую фазу, в которой вследствие пьезоэффекта происходит изменение поляризации, что приводит к возникновению напряжения на обкладках конденсатора. При инверсном МЭ-эффекте в результате подачи напряжения на обкладки конденсатора в пьезоэлектрической компоненте возникают деформации, которые передаются в магнитострикционную фазу, где, вследствие магнитострикции, происходит изменение намагниченности, что приводит к возникновению ЭДС индукции на катушке, внутри которой находится образец. С точки зрения практического применения инверсный МЭ-эффект интересен тем, что на его основе можно создавать трансформаторы, имеющие, в отличие от классических аналогов, всего одну обмотку $[15,16]$.

При теоретическом описании МЭ-эффекта часто используется метод эффективных параметров [17,18]. Однако этот метод применим тогда, когда характерные размеры композита много меньше длины звуковой волны. Он хорошо подходит для описания МЭ-эффекта в объемных композитах. Описание инверсного МЭ-эффекта на основе этого метода выполнено в работах [19-21]. Однако для слоистых магнитострикционнопьезоэлектрических структур, когда длина звуковой волны соизмерима с толщиной слоев, этот метод неприменим. Кроме того, большая трудность заключатся в расчете самих эффективных параметров. Более точным методом является метод, основанный на решении уравнений эластодинамики и электростатики отдельно для пьезоэлектрической и магнитострикционной фаз при учете условий на границе раздела между фазами [22,23]. Ранее, в работах [24-26], была представлена теория инверсного МЭ-эффекта, основанная на решении уравнений эластодинамики и электростатики отдельно для магнитострикционной и пьезоэлектрической фазы с учетом условий на границе раздела. Однако в данных работах предполагалось, что смещения и деформации магнитострикционного и пьезоэлектрического слоев одинаковы и граница раздела между слоями никак не учитывалась. При инверсном МЭ-эффекте под действием электрического поля колебания среды сначала возбуждаются в пьезоэлектрическом слое. Затем, посредством тангенциальных напряжений, эти колебания передаются через границу раздела в магнетик, где, вследствие магнитострикции, происходит изменение намагниченности. При распространении колебаний по толщине образца, вследствие того, что магнитная фаза является пассивной по отношению к электрическому полю, которое возбуждает колебания в пьезоэлектрике, происходит изменение амплитуды колебаний среды, вследствие чего деформации и напряжения в магнетике и пьезоэлектрике будут неоднородными по толщине образца. В данной работе этот механизм возбуждения колебаний учтен в явном виде путем введения граничных условий на интерфейсе между фазами, что делает модель более точной.

\section{2. Инверсный эфффект в двухслойной пластине}

При инверсном эффекте в качестве объекта исследований используют образец из магнитоэлектрического материала, помещенного внутрь катушки с числом витков $N$. Схематичное изображение такой структуры приведено на рис. 1.

Для теоретического описания эффекта в качестве модели рассмотрим образец в виде прямоугольной пластинки длиной $L$ и шириной $W$, представляющий собой двухслойную магнитострикционно-пьезоэлектрическую структуру с толщиной магнетика $t_{m}$ и пьезоэлектрика $t_{p}$ (рис. 2). На верхней и нижней поверхности пластинки нанесены тонкие металлические электроды. Будем считать пластинку тонкой и узкой, т.е. $L \gg W, t_{m}, t_{p}$. Это позволяет ограничиться рассмотрением колебаний, распространяющихся только по длине пластинки, вследствие того, что колебания, распространяющиеся по толщине и ширине пластинки, будут более высокочастотными. Образец предварительно поляризован в направлении перпендикулярно слоям пластинки. Начало системы координат совместим с границей раздела слоев, а ось $Z$ направим вертикально вверх, перпендикулярно

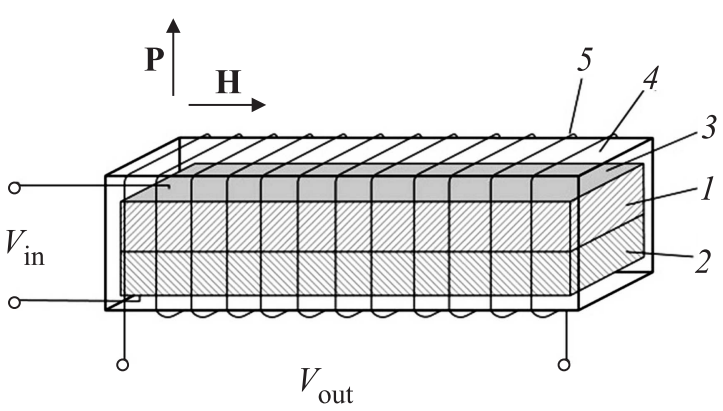

Рис. 1. Схематичное изображение структуры. 1 - магнетик, 2 - пьезоэлектрик, 3 - электроды, 4 - непроводящий каркас, 5 - обмотка катушки.

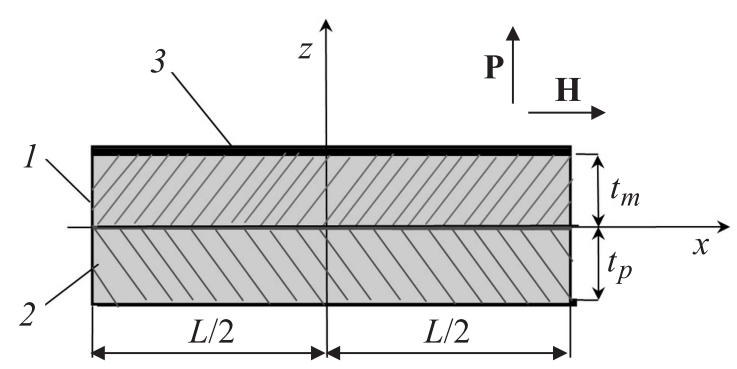

Рис. 2. Модель двухслойной структуры. 1 - магнетик, 2 - пьезоэлектрик, 3 - электроды. 
слоям. Ограничимся рассмотрением поперечного эффекта вследствие того, что он гораздо легче реализуется в эксперименте, чем продольный. В этом случае поле подмагничивания $\mathbf{H}_{\text {bias }}$ направлено по оси $X$ перпендикулярно вектору поляризации Р. Рассмотрим планарные колебания, которые распространяются вдоль оси $X$. Переменное электрическое поле с частотой $\omega$ возбуждает в пьезоэлектрической подсистеме упругие колебания, которые посредством касательных напряжений передаются через границу раздела в магнитострикционный слой, в результате чего происходит изменение намагниченности.

Поскольку пластинка тонкая и узкая, то отличными от нуля компонентами тензора напряжений будут только ${ }^{a} T_{x x}$ и ${ }^{a} T_{x z}$, где индекс $\alpha=p$ для пьезоэлектрика и $\alpha=m$ для магнетика. При данной ориентации полей уравнения тензора деформаций для пьезоэлектрической и магнитострикционной фаз ${ }^{\alpha} S_{i j}$ и $x-$ проекция вектора магнитной индукции ${ }^{m} B_{x}$ имеют следующий вид

$$
\begin{gathered}
{ }^{p} S_{x x}=\frac{1}{Y_{p}}{ }^{p} T_{x x}+d_{x x, z} E_{z}, \\
{ }^{p} S_{x z}=\frac{1}{G_{p}}{ }^{p} T_{x z} .
\end{gathered}
$$

Здесь ${ }^{p} T_{x x}$ и ${ }^{p} T_{x z}$ - компоненты тензора напряжений в пьезоэлектрической фазе, $Y_{p}, G_{p}-$ модуль Юнга и модуль сдвига пьезоэлектрика, $d_{x x, z}$ - пьезоэлектрический тензор, $E_{z}-z$-проекция вектора напряженности электрического поля.

Для магнитострикционной фазы аналогичные уравнения запишутся в следующей форме

$$
\begin{gathered}
{ }^{m} S_{x x}=\frac{1}{Y_{m}}{ }^{m} T_{x x}+q_{x x, x}^{B} B_{x}, \\
{ }^{m} S_{x z}=\frac{1}{G_{m}}{ }^{m} T_{x z}, \\
B_{x}=\mu_{x x}\left(H_{x}+q_{x x, x}^{B}{ }^{m} T_{x x}\right),
\end{gathered}
$$

где ${ }^{m} T_{x x}$ и ${ }^{m} T_{x z}$ - компоненты тензора напряжений в магнитострикционной фазе, $Y_{m}, G_{m}-$ модуль Юнга и модуль сдвига магнетика, $q^{B}=\frac{\partial \lambda}{\partial B}-$ пьезомагнитный коэффициент, $H_{x}$ - напряженность магнитного поля, $\lambda$ - магнитострикция магнетика.

Уравнение движения для смещений среды имеет вид

$$
\rho_{\alpha} \frac{\partial^{2 \alpha} u_{x}}{\partial t^{2}}=\frac{\partial^{\alpha} T_{x x}}{\partial x}+\frac{\partial^{\alpha} T_{x z}}{\partial z}
$$

где $\rho_{\alpha}-$ плотность магнетика или пьезоэлектрика, ${ }^{\alpha} T_{i j}$ - тензор напряжений.

Как и в [17], решение уравнения для вектора смещения среды (6) представим в виде плоских волн, амплитуда которых изменяется по толщине образца

$$
{ }^{\alpha} u(x, z)={ }^{\alpha} g(z)\left(A_{1} \cos (\omega t-k x)+A_{2} \sin (\omega t-k x)\right),
$$

где $A_{1}$ и $A_{2}$ - постоянные интегрирования, ${ }^{\alpha} g(z)-$ некоторая функция. Для нахождения этой функции под- ставим выражение (7) в уравнение (6), в результате чего получим два дифференциальных уравнения для функций ${ }^{p} g(z)$ и ${ }^{m} g(z)$ с четырьмя неизвестными постоянными интегрирования, которые определяются с помощью граничных условий. На границе раздела в точке $z=0$ равны смещения сред и также равны тангенциальные составляющие тензора напряжений, т.е. имеют место следующие равенства

$$
{ }^{p} u(x, 0)={ }^{m} u(x, 0) ; \quad{ }^{p} T_{x z}(x, 0)={ }^{m} T_{x z}(x, 0) .
$$

На свободных верхней и нижней поверхностях образца тангенциальные компоненты тензора напряжений равны нулю, т.е. имеют место следующие граничные условия

$$
{ }^{p} T_{x z}\left(x, z=t_{p}\right)=0 \quad \text { и } \quad{ }^{m} T_{x z}\left(x, z=-t_{m}\right)=0 .
$$

Используя граничные условия $(8,9)$, получим систему уравнений, условие совместности которой приводит к следующему уравнению

$$
Y_{m} \chi_{m} \operatorname{th}\left(\kappa_{m}\right)=Y_{p} \chi_{p} \operatorname{tg}\left(\kappa_{p}\right)
$$

где $\kappa_{m}=\chi_{m} t_{m}$ и $\chi_{p}=\chi_{p} t_{p}-$ безразмерные переменные. Здесь введены обозначения

$$
\begin{gathered}
\chi_{m}^{2}=-2(1+v)\left[\frac{\omega^{2}}{V_{m}^{2}}-k^{2}\right], \quad \chi_{p}^{2}=2(1+v)\left[\frac{\omega^{2}}{V_{p}^{2}}-k^{2}\right], \\
V_{m}=\sqrt{\frac{Y_{m}}{\rho_{m}}}, \quad V_{p}=\sqrt{\frac{Y_{p}}{\rho_{p}}}
\end{gathered}
$$

- скорости продольных волн в магнетике и пьезоэлектрике соответственно.

Соотношение (10) в неявном виде определяет зависимость угловой частоты $\omega$ от волнового вектора $k$ и в таком виде не совсем удобно для практического применения. В это уравнение входят два параметра $\kappa_{m}$ и $\kappa_{p}$, которые для реальных структур являются малыми. Разлагая уравнение (10) в ряд по этим параметрам и учитывая лишь первые неисчезающие члены, для дисперсионного соотношения получим дисперсионное соотношение в виде

$$
\omega=\bar{V}(1+\delta) k
$$

где $\bar{V}=\sqrt{\frac{\overline{\bar{Y}}}{\bar{\rho}}}-$ скорость распространения колебаний в структуре с усредненными параметрами, $\delta$ - поправка, описывающая отклонение от линейного соотношения между $\omega$ и $k$. Здесь $\bar{Y}=\left(Y_{m} t_{m}+Y_{p} t_{p}\right) /\left(t_{m}+t_{p}\right)$, $\bar{\rho}=\left(\rho_{m} t_{m}+\rho_{m} t_{m}+\rho_{p} t_{p}\right) /\left(t_{m}+t_{p}\right)-$ средние значения модуля Юнга и плотности структуры.

С учетом первых неисчезающих членов поправка $\delta$ определяется следующим выражением

$$
\begin{aligned}
\delta & =-\frac{1+v}{3} \\
& \times \frac{Y_{m} t_{m}\left[\left(\bar{V} / V_{m}\right)^{2}-1\right]^{2}\left(k t_{m}\right)^{2}+Y_{p} t_{p}\left[\left(\bar{V} / V_{p}\right)^{2}-1\right]^{2}\left(k t_{p}\right)^{2}}{Y_{m} t_{m}+Y_{p} t_{p}} .
\end{aligned}
$$


Выполняя вычисления, аналогичные вычислениям, проведенным в работе [23], для компонент тензора напряжений в магнитострикционной фазе получим уравнения в виде

$$
\begin{aligned}
{ }^{m} T_{x x}= & Y_{m}\left[k A _ { 2 } \operatorname { c o s } ( k x ) \left(\exp \left(-2 \kappa_{m}\right) \exp \left(\chi_{m} z\right)\right.\right. \\
& \left.\left.+\exp \left(-\chi_{m} z\right)\right)-g_{x x, x}^{B} B_{x}\right],
\end{aligned}
$$

где

$$
A_{2}=\frac{\gamma q_{x x, x}^{B} B_{x}+d_{x x, x} E_{z}}{k \cos (\kappa)\left(1+\exp \left(-2 \kappa_{m}\right)\right)\left(\gamma \frac{\operatorname{th}\left(\kappa_{m}\right)}{\kappa_{m}}+\frac{\operatorname{tg}\left(\kappa_{p}\right)}{\kappa_{p}}\right)}
$$

и введены безразмерные параметры $\kappa=k L / 2$, $\gamma=Y_{m} t_{m} / Y_{p} t_{p}$.

Коэффициент инверсного МЭ преобразования структуры определим как отношение среднего значения индукции магнитного поля в образце к величине приложенного внешнего электрического поля, ее вызвавшей, T. e.

$$
\alpha_{B}=\frac{\left\langle B_{x}\right\rangle}{\left\langle E_{z}\right\rangle}=\frac{1}{L \cdot t_{m}} \frac{\int_{0}^{t_{m}} \int_{-L / 2}^{L / 2} B_{x}(x, z) d x d z}{E_{z}} .
$$

Подставляя выражение для тензора напряжений (13) в уравнение для магнитной индукции (5) и выполняя интегрирование с учетом закона полного тока и условия разомкнутой цепи, для коэффициента инверсного МЭ преобразования получим выражение в виде

$$
\alpha_{B}=Y_{m} \mu_{x x} q_{x x, x}^{B} d_{x x, x} \frac{\Delta}{\Delta_{a}} .
$$

Здесь введены следующие обозначения

$$
\begin{aligned}
\Delta & =\frac{\operatorname{tg}(\kappa)}{\kappa} \cdot \frac{\operatorname{th}\left(\kappa_{m}\right)}{\kappa_{m}} /\left(\gamma \frac{\operatorname{th}\left(\kappa_{m}\right)}{\kappa_{m}}+\frac{\operatorname{tg}\left(\kappa_{p}\right)}{\kappa_{p}}\right), \\
\Delta_{a} & =1+K_{m}^{2}(1-\gamma \Delta), \quad K_{m}^{2}=Y_{m} \mu_{x x}\left(q_{x x, x}^{B}\right)^{2} .
\end{aligned}
$$

Как следует из (15), при значении параметра $\Delta_{a}=0$ имеет место резонансное увеличение эффекта.

В области низких частот инверсный МЭ коэффициент практически не зависит от частоты. В предельном случае низких частот, разлагая в ряд уравнение (15) по малым параметрам $\kappa, \kappa_{m}, \kappa_{p}$ и ограничиваясь первыми членами разложения, для его низкочастотного значения получим выражение в следующем виде

$$
\alpha_{B}=Y_{m} \mu_{x x} q_{x x, x}^{B} d_{x x, z} /\left(1+\gamma+K_{m}^{2}\right) .
$$

Выходное напряжение, индуцируемое на катушке, найдем, используя закон Фарадея для электромагнитной индукции. Полагая, что все магнитное поле сосредоточено в магнетике, для индуцированного напряжения получим выражение в виде

$$
V_{\mathrm{inv}}=\omega \alpha_{B} \frac{t_{m}}{t_{p}} W N V_{\mathrm{in}}
$$

где $V_{\text {in }}$ - входное напряжение, подаваемое на обкладки структуры, $V_{\text {out }}$ - выходное напряжение, снимаемое с катушки индуктивности.
Как следует из (19), коэффициент передачи напряжения определяется выражением

$$
K_{T}=\omega \alpha_{B} \frac{t_{m}}{t_{p}} W N
$$

Его зависимость от частоты также имеет резонансный характер, а численное значение определяется не только характеристиками образца, но и числом витков катушки.

\section{3. Трехслойная структура}

На практике для увеличения эффективности преобразования наряду с двухслойными структурами [25,28,29] часто используются трехслойные структуры [24,27]. Схематичное изображение такой структуры представлено на рис. 3. Использование симметричной трехслойной структуры позволяет более эффективно возбудить в магнетике планарные колебания и исключить изгибные моды.

В силу симметрии задачи начало системы координат совместим с серединой магнетика, а ось $Z$ направим вертикально вверх, перпендикулярно слоям. Уравнения движения и уравнения для тензора напряжений для магнитострикционной и пьезоэлектрической фаз будут по прежнему определяться уравнениями (1-6). Однако граничные условия запишутся в несколько ином виде. На границе раздела слоев, т.е. в точках $z= \pm t_{m} / 2$, будут равны смещения сред и тангенциальные напряжения, т. е. ${ }^{p} u\left(x, \pm t_{m} / 2\right)={ }^{m} u\left(x, \pm t_{m} / 2\right)$, ${ }^{p} T_{x z}\left(x, \pm t_{m} / 2\right)={ }^{m} T_{x z}\left(x, \pm t_{m} / 2\right)$, а на верхней и нижней свободных поверхностях пьезоэлектрика будет равенство нулю тангенциальных напряжений, т.е. ${ }^{p} T_{x z}\left(x, z= \pm\left(t_{p}+t_{m} / 2\right)\right)=0$.

Возбуждение колебаний в магнетике осуществляется пьезоэлектриком посредством передачи колебаний через границу раздела. Поскольку возбуждение колебаний происходит симметрично относительно начала координат, то очевидно, что в центре магнетика, т.е. в точке $z=0$ амплитуда колебаний будет иметь экстремум, что означает $\frac{\partial^{m} u(x, z)}{\partial z}=0$ при $z=0$. Учитывая взаимосвязь между тангенциальной компонентой тензора напряжений и

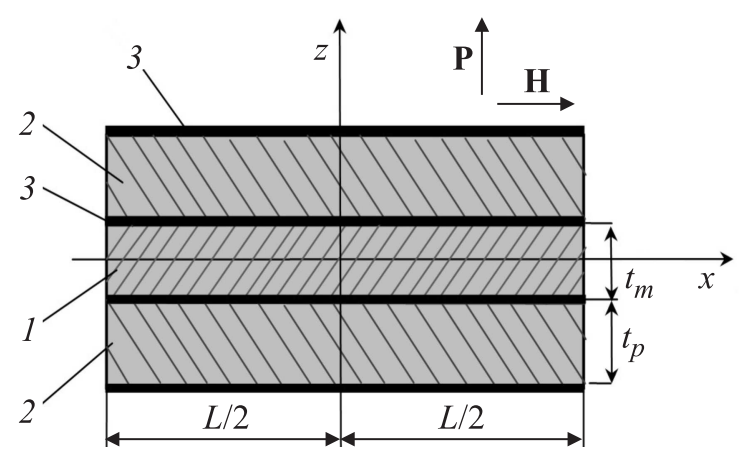

Рис. 3. Модель трехслойной структуры пьезоэлектрик-магнетик-пьезоэлектрик. 1 - магнетик, $2-$ пьезоэлектрик, 3 - электроды. 
деформацией сдвига, равенство нулю производной дает равенство нулю тангенциальной компоненты тензора напряжений в точке $z=0$. Таким образом, задача о трехслойной структуре сводится к задаче о двухслойной структуре с толщиной пьезоэлектрика $t_{p}$ и толщиной магнетика, равной $t_{m} / 2$.

\section{4. Сравнение с экспериментом}

В области низких частот индуцируемое вследствие инверсного МЭ-эффекта напряжение на катушке $V_{\text {out }}$ и коэффициент передачи напряжения линейно возрастает с частотой, но по мере приближения к области электромеханического резонанса зависимость становится нелинейной. На рис. 4 представлена рассчитанная по формуле (19) зависимость индуцированного напряжения $V_{\text {inv }}$ от частоты приложенного электрического поля для структуры $\mathrm{PZT}-\mathrm{Ni}-\mathrm{PZT}$ в области электромеханического резонанса, а на рис. 5- в низкочастотной области спектра. При расчетах использовались следующие параметры: длина $L=9.5 \mathrm{~mm}$, ширина $W=4.5 \mathrm{~mm}$, толщина каждого слоя пьезоэлектрика $t_{p}=0.5 \mathrm{~mm}$, толщина никеля $t_{m}=0.2 \mathrm{~mm}$, модули Юнга $Y_{m}=210 \mathrm{GPa}$ и $Y_{p}=67 \mathrm{GPa}$ для $\mathrm{Ni}$ и $\mathrm{PZT}$ соответственно, $\rho_{m}=8900 \mathrm{~kg} / \mathrm{m}^{3}$ и $\rho_{p}=7000 \mathrm{~kg} / \mathrm{m}^{3}$ их плотности. Значение пьезомодуля принималось равным $d_{z, x x}=-150 \mathrm{~m} / \mathrm{V}$, величина пьезомагнитного коэффициента $q_{x x, x}^{B}$ определялась из результатов работы [30]. Как видно из рисунка, в области низких частот наблюдается линейная зависимость $V_{\text {out }}$ от частоты, а на частоте порядка $180 \mathrm{kHz}$ наблюдается резонансное увеличение эффекта. Рассчитанные теоретические частотные зависимости $V_{\text {out }}$ хорошо согласуется с экспериментальными данными, представленными в работе [24].

На рис. 6 представлена рассчитанная частотная зависимость коэффициента инверсного МЭ-преобра-

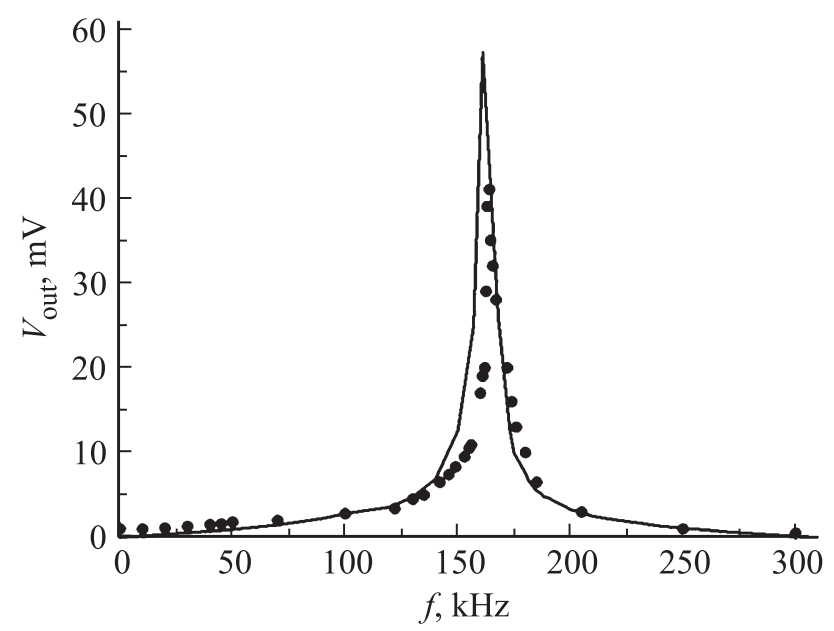

Pис. 4. Зависимость индуцированного напряжения $V_{\text {out }}$ от частоты приложенного электрического поля для структуры $\mathrm{PZT}-\mathrm{Ni}-\mathrm{PZT}$ в области электромеханического резонанса. Сплошная линия - теория, точки - эксперимент [21].

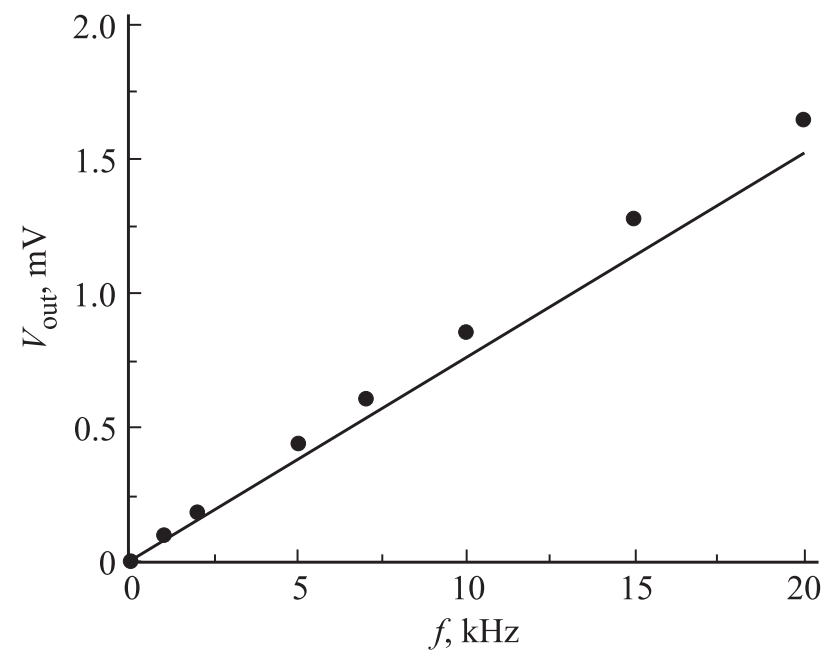

Рис. 5. Зависимость индуцированного напряжения $V_{\text {out }}$ от частоты приложенного электрического поля для структуры $\mathrm{PZT}-\mathrm{Ni}-\mathrm{PZT}$ в низкочастотной области. Сплошная линия теория, точки - эксперимент [21].

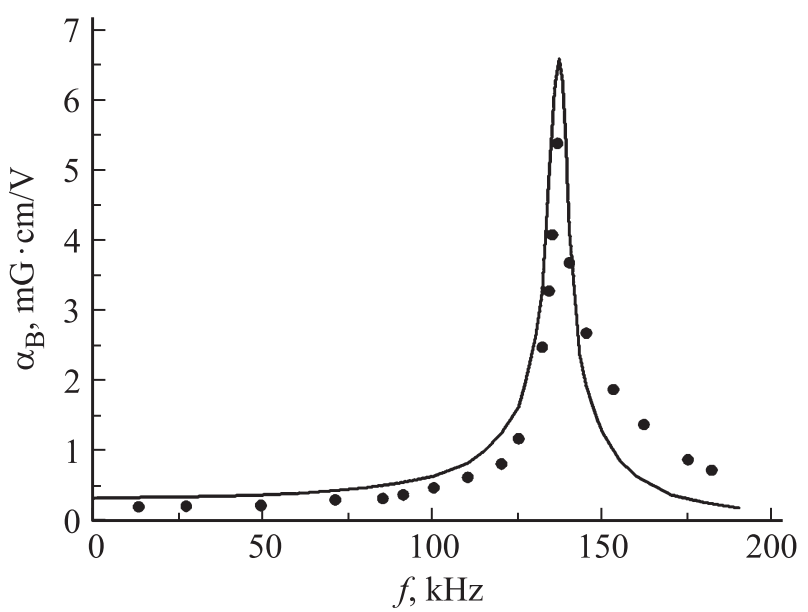

Рис. 6. Частотная зависимость коэффициента инверсного МЭ-преобразования $\alpha_{B}$ от частоты для структуры $D$-терфенол-PZT. Сплошная линия - теория, точки - эксперимент [26].

зования $\alpha_{B}$ от частоты для структуры $D$-терфенол-PZT. При расчетах использовались параметры структуры, представленные в работе [29]. Параметры $D$-терфенола, используемые в расчетах, брались из работы [31]. Как видно из рисунка, также имеет место пиковое увеличение эффекта на частоте электромеханического резонанса.

\section{5. Заключение}

Таким образом, как следует из представленных выше выражений для величин, характеризующих инверсный МЭ-эффект, в области низких частот коэффициент инверсного МЭ-преобразования не зависит от частоты, в 
то время как индуцированное на катушке индуктивности напряжение и коэффициент передачи напряжения линейно возрастают с увеличением частоты. По мере приближения к области электромеханического резонанса зависимость указанных величин становится нелинейной и на частоте электромеханического резонанса наблюдается пиковое увеличение эффекта. Частота электромеханического резонанса зависит не только от длины образца, но также от толщины магнитострикционного и пьезоэлектрического слоев. Величина коэффициента инверсного МЭ-преобразования нелинейным образом зависит от толщины магнитострикционного и пьезоэлектрического слоев, причем кривая имеет максимум, положение которого зависит от характеристик магнитострикционного и пьезоэлектрического слоев. Представленные в работе теоретические зависимости хорошо согласуются с экспериментальными данными.

\section{Список литературы}

[1] Л.Д. Ландау, Е.М. Лифшиц. Электродинамика сплошных сред. М. ГИФМЛ (1959). 532 с.

[2] И.Е. Дзялошинский. ЖЭТФ 37, 881 (1959).

[3] Д.Н. Астров. ЖЭТФ 40, 1035 (1961).

[4] V.J. Folen, G.T. Rado, E.W. Stalder. Phys. Rev. Lett. 6, 607 (1961).

[5] А.П. Пятаков, А.К. Звездин. УФН 182, 593 (2012).

[6] E. Kita, K. Siratori, A.J. Tasaki. J. Phys. Soc. Jpn. 46, 1033 (1979).

[7] М.И. Бичурин, В.М. Петров. ФТТ 29, 2509 (1987).

[8] G. Srinivasan, Y.K. Fetisov. Integrated Ferroelectrics 83, 89 (2006).

[9] H-m. Zhou, O. Chen, J.-H. Deng. Chin. Phys. B 23, 04752 (2014).

[10] M.I. Bichurin, D.A. Filippov. Ferroelectrics 204, 225 (1997).

[11] J. Van den Boomgard, R.A.J. Born, H.F.J.I. Giller. J. Mater. Sci. 9, 1705 (1974).

[12] A.M.J.G. Van Run, D.R. Terrell, J.H. Scholing. J. Mater. Sci. 9, 1710 (1974).

[13] C.W. Nan, M.I. Bichurin, S. Dong, D. Viehland, G. Srinivasan. J. Appl. Phys. 103, 031101 (2008).

[14] Y. Wang, J. Li, D. Vieland. Mater. Today 17, 269 (2014).

[15] S. Dong, J.F. Li, D. Viehland, J. Cheng, L.E. Cross. Appl. Phys. Lett. 83, 1354 (2004).

[16] Д.А. Филиппов, Т.А. Галкина, В.М. Лалетин, G. Srinivasan. Письма в ЖТФ 38, 82 (2012).

[17] G. Harshe, J.O. Dougherty, R.E. Newnham. Int. J. Appl. Electromagn. Mater. 4, 145 (1993).

[18] M.I. Bichurin, V.M. Petrov, G. Srinivasan. Phys. Rev. B 68, 054402 (2003).

[19] Д.А. Филиппов, Т.А. Галкина, G. Srinivasan. Письма в ЖТФ 36, 23 (2010).

[20] Д.А. Филиппов, Т.А. Галкина, В.М. Лалетин, G. Srinivasan. ФTT 53, 1737 (2011).

[21] Д.А. Филиппов, В.М. Лалетин, Г.С. Радченко. Письма в ЖТФ 41, 91 (2015).

[22] Д.А. Филиппов. ФТТ 47, 1082 (2005).

[23] Д.А. Филиппов, В.М. Лалетин, Т.А. Galichyan. ФТТ 55, 1728 (2013).
[24] Y.K. Fetisov, V.M. Petrov, G. Srinivasan. J. Mater. Res. 22, 2074 (2007).

[25] B. Tong, X.F. Yang, J. Ouyang, G.Q. Lin, S. Chen. J. Alloys Comp. 563, 51 (2013).

[26] M.I. Bichurin, V.M. Petrov, R.V. Petrov. J. Magn. Magn. Mater. 324, 3548 (2012).

[27] H.C. Xuan, L.Y. Wang, S.C. Ma, Y.X. Zheng, Q.Q. Cao, D.H. Wang, Y. W. Du. Appl. Phys. Lett. 98, 052505 (2011).

[28] Yuan Zhang, Guoxi Liu, Meiya Li, Jun Li, Yongdan Zhu. J. Alloys Comp. 641, 188 (2015).

[29] А.В. Калгин, С.А. Гриднев, Z.Н. Gribe. ФТТ 56, 1278 (2014).

[30] D.A. Burdin, D.V. Chashin, N.A. Ekonomov, L.Y. Fetisov, Y.K. Fetisov, G. Srinivasan, G. Sreenivasulu. J. Magn. Magn. Mater. 358-59, 98 (2014).

[31] S.H. Lim, S.R. Kim, S.Y. Kang, J.K. Park, J.T. Nam, D. Son. J. Magn. Magn. Mater. 191, 113 (1999). 